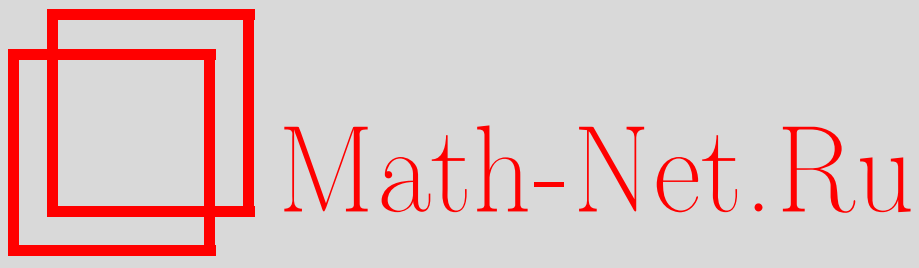

А. Ф. Дюмин, Оптимальный линейный фильтр в задаче калибровки погрешностей инерциальных измерителей угловой скорости, Вестн. Сам. гос. техн. ун-та. Сер. Физ.-мат. науки, 2006, выпуск 42, 169-173

DOI: https://doi.org/10.14498/vsgtu428

Использование Общероссийского математического портала Math-Net.Ru подразумевает, что вы прочитали и согласны с пользовательским соглашением

http://www.mathnet.ru/rus/agreement

Параметры загрузки:

IP: 52.23 .180 .231

26 апреля 2023 г., 06:26:26

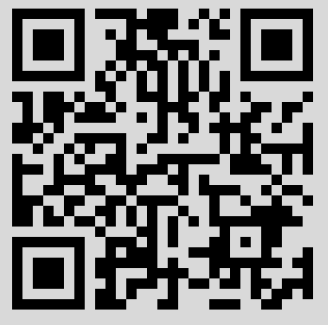




\section{ОПТИМАЛЬНЫЙ ЛИНЕЙНЫЙ ФИЛЬТР В ЗАДАЧЕ КАЛИБРОВКИ ПОГРЕШНОСТЕЙ ИНЕРЦИАЛЬНЫХ ИЗМЕРИТЕЛЕЙ УГЛОВОЙ СКОРОСТИ}

Рассматривается задача калибровки мультипликативных погрешностей датчиков угловой скорости, входящих в состав бесплатформенной инерцилальной системы определения ориентации низкоорбитального космического аппарата в качестве первичных измерителей. Показано, что в этой задаче можно использовать результаты теории оптимальной линейной фильтрации КалманаБьюси. Получено аналитическое решение матричного дифференциального уравнения Риккати, инвариантное по отношению к закону изменения угловой скорости калибровочного маневра космического аппарата, что позволяет осуществить параметрический синтез оптимального фильтра.

Точность определения ориентации низкоорбитальных космических аппаратов во многом зависит от точности первичных измерителей кинематических параметров углового движения. Современные бесплатформенные инерциальные системы определения ориентации основаны на интегрировании кинематических уравнений углового движения твердого тела, при этом в качестве первичных измерителей используются датчики угловой скорости. В [1] рассмотрена задача синтеза оптимальных устройств наблюдения, предназначенных для высокоточной оценки угловой скорости космического аппарата относительно инерциальной системы координат по сигналам датчиков угловой скорости или датчиков угла гиростабилизированной платформы. При этом оцениваются и компенсируются в основном постоянная и шумовая составляющие аддитивной погрешности датчиков угловой скорости. Для этой цели используются динамические уравнения углового движения космического аппарата. Однако существенное значение имеет и оценка мультипликативных погрешностей этих датчиков, обусловленных вариацией крутизны выходного сигнала датчика, неортогональностью осей чувствительности датчиков угловой скорости, погрешностями установки блока инерциальных измерителей в корпусе космического аппарата и т.п. Для оценки этих погрешностей могут быть использованы специальные калибровочные маневры космического аппарата, проводимые во время его полета, в результате которых можно оценить указанные погрешности путем использования измерительной информации, получаемой с помощью астроориентаторов $[2,3]$. При этом алгоритмы калибровки рассматриваемых погрешностей предполагают дискретную обработку измерительной информации, в частности, использование результатов астровизирования, полученных в начале и в конце калибровочных маневров.

Целью настоящей работы является синтез алгоритма наблюдения мультипликативных погрешностей датчиков угловой скорости по результатам непрерывных астроизмерений.

Предполагается, что постоянные уходы гироскопов датчиков угловой скорости могут быть оценены и компенсированы, а влиянием неортогональности измерительных осей этих датчиков можно пренебречь. Кроме того, предполагается, что астроориентатор работоспособен при проведении калибровочных разворотов космического аппарата.

Введем в рассмотрение следующие координатные базисы [4] (все правые, ортогональные): I - инерциальный опорный базис, относительно которого определяется ориентация космического аппарата (с помощью бесплатформенной инерциальной системы ориентации) и в котором заданы координаты звездных ориентиров (с помощью звездного каталога); $\mathbf{E}-$ связанный с космическим аппаратом базис, ориентация которого в базисе I определяется кватернионом $\Lambda ; \mathbf{B}_{\Gamma}-$ базис измерительных осей бесплатформенной инерциальной системы ориентации, в проекциях на оси которого чувствительные элементы системы ориентации воспринимают абсолютную угловую скорость космического аппарата; положение базиса $\mathbf{B}_{\Gamma}$ относительно базиса $\mathbf{E}$ определяется вектором малого поворота $\bar{\beta}$, координаты которого можно интерпретировать как погрешности установки блока чувствительных элементов системы ориентации относительно базиса $\mathbf{E} ; \mathbf{E}_{T}$ - координатный базис, соответствующий вычисленному с помощью бесплатформенной инерциальной системы ориентации положению связанного с космическим аппаратом базиса, ориентация которого в базисе $\mathbf{I}$ определяется кватернионом $\Lambda_{\Gamma} ; \mathbf{E}_{A}-$ связанный базис, ориентация которого определяется с помощью астроориентатора, формирующего кватернион $\Lambda_{A}$. 
Связь между кватернионами $\Lambda, \Lambda_{\Gamma}, \Lambda_{A}$ с точностью до малых первого порядка малости определяется уравнениями:

$$
\begin{gathered}
\Lambda_{\Gamma}=\Lambda \mathrm{o}\left(1+\frac{1}{2} \Delta \bar{\Theta}\right), \\
\Lambda_{A}=\Lambda \mathrm{o}\left(1+\frac{1}{2} \bar{\gamma}\right),
\end{gathered}
$$

где $\Delta \bar{\Theta}$ - вектор малого поворота реального базиса $\mathbf{E}$ при переходе к базису, вычисленному с помощью бесплатформенной инерциальной системы ориентации, т.е. вектор погрешностей этой системы; $\bar{\gamma}$ - вектор малого поворота, обусловленного погрешностями установки астроориентатора относительно базиса $\mathbf{E}$.

Дифференциальное уравнение для вектора ошибки $\Delta \bar{\Theta}$ бесплатформенной инерциальной системы ориентации имеет вид [5]:

$$
\Delta \Theta=-[\omega \times] \Delta \Theta+\delta \bar{\omega}
$$

где $[\omega \times]=\left\|\begin{array}{ccc}0 & -\omega_{z} & \omega_{y} \\ \omega_{z} & 0 & -\omega_{x} \\ -\omega_{y} & \omega_{x} & 0\end{array}\right\|-$ кососимметрическая матрица, элементами которой являются проекции вектора $\bar{\omega}$ абсолютной угловой скорости космического аппарата на связанный базис; $\delta \bar{\omega}$ - погрешность первичных измерителей бесплатформенной инерциальной системы ориентации, представляющая собой разность между измеренной угловой скоростью $\bar{\omega}_{u}$ и истинной угловой скоростью $\bar{\omega}$.

С учетом введенного вектора малого поворота $\bar{\beta}$, определяющего взаимное положение базисов $\mathbf{B}_{\Gamma}$ и $\mathbf{E}$, можно показать, что:

$$
\bar{\omega}_{u}=\left(\mathbf{E}_{3}-[\beta \times]\right) \bar{\omega},
$$

где $\mathbf{E}_{3}$ - единичная 3х3-матрица; $[\beta \times]$ - матрица такой же структуры, что и матрица $[\omega \times]$, в которой вектор $\bar{\omega}$ заменен на вектор $\bar{\beta}$. Тогда, используя структуру матриц $[\omega \times]$ и $[\beta \times]$, можно получить следующее выражение для $\delta \bar{\omega}$ :

$$
\delta \bar{\omega}=[\omega \times] \bar{\beta} .
$$

Подставляя (4) в (3) и вводя вектор малого поворота $\bar{\alpha}=\Delta \bar{\Theta}-\bar{\beta}$ как угол между базисом $\mathbf{E}_{\Gamma}$ и базисом $\mathbf{B}_{\Gamma}$, будем иметь:

$$
£=-[\omega \times] \bar{\alpha} .
$$

По имеющейся на борту информации об ориентации космического аппарата (кватернионы $\Lambda_{A}$ и $\Lambda_{\Gamma}$ ) может быть сформирован вектор измерений в виде:

$$
\overline{\mathrm{y}}=2 \operatorname{vect}\left(\kappa_{A}^{\circ} \mathrm{\circ} \Lambda_{\Gamma}\right),
$$

где через $K_{A}^{\circ}$ обозначен кватернион, сопряженный по отношению к кватерниону $\Lambda_{A}$.

Подставляя (1), (2) в выражение (6), с точностью до слагаемых первого порядка малости будем иметь:

$$
\overline{\mathrm{y}}=\bar{\alpha}+\bar{\beta}-\bar{\gamma} .
$$

Таким образом, вектор измерений $\overline{\mathbf{y}}$ характеризует вектор малого поворота базиса $\mathrm{E}_{\Gamma}$ относительно базиса $\mathbf{E}_{A}$, т.е. базиса, вычисленного с помощью бесплатформенной инерциальной системы ориентации, относительно базиса, связанного с астроориентатором.

Из выражения (7) видно, что оценка погрешности $\bar{\alpha}$ установки измерительных осей бесплатформенной инерциальной системы ориентации относительно связанного базиса $\mathbf{E}$ возможна лишь с точностью до постоянной аддитивной погрешности $\bar{\beta}-\bar{\gamma}$.

Введем в рассмотрение вектор $\bar{\delta}=\bar{\beta}-\bar{\gamma}$. Для него может быть записано дифференциальное уравнение формирующего фильтра:

$$
\delta=\mathbf{0} .
$$


Тогда уравнения (5), (8) можно рассматривать как уравнения состояния объекта наблюдения, а уравнение (7), с учетом шумовой составляющей погрешности измерения $\bar{\eta}$, переписанное в виде:

$$
\bar{y}=\bar{\alpha}+\bar{\delta}+\bar{\eta},
$$

как уравнение системы измерения.

Таким образом, задача оценки вектора погрешности $\bar{\alpha}$ сводится к задаче оценки состояния динамического объекта наблюдения (5), (8) по сигналу системы измерения (9)

Для решения такого рода задачи можно, в частности, использовать теорию оптимальной линейной фильтрации Калмана-Бьюси [6, 7].

Вектор состояния объекта наблюдения может быть представлен в виде:

$$
\mathbf{x}=\left\|\begin{array}{ll}
\bar{\alpha}^{T} & \bar{\delta}^{T}
\end{array}\right\|^{T} .
$$

Тогда уравнения (5), (8) можно переписать в виде одного векторно-матричного уравнения

$$
\hat{x}=\mathbf{A x}
$$

где $\mathbf{A}=\left\|\begin{array}{cc}-[\omega \times & \mathbf{0}_{3 \times 3} \\ \mathbf{0}_{3 \times 3} & \mathbf{0}_{3 \times 3}\end{array}\right\|, \mathbf{0}_{3 \times 3}-$ нулевая матрица с размерностями 3х3, а уравнение (9) - в виде:

$$
\overline{\mathbf{y}}=\mathbf{C x}+\bar{\eta},
$$

где $\mathbf{C}=\left\|\mathbf{E}_{3} \quad \mathbf{E}_{3}\right\|$

Предполагается, что шумовая составляющая погрешности измерения $\bar{\eta}$ аппроксимируется гауссовским белым шумом с заданной интенсивностью.

Состояние объекта наблюдения (10) по сигналу системы измерения (11) может быть оценено с помощью оптимального линейного фильтра Калмана-Бьюси, структура которого определяется векторно-матричным дифференциальным уравнением

$$
\hat{\mathbf{x}}=\mathbf{A} \hat{\mathbf{x}}+\mathbf{K}(\overline{\mathbf{y}}-\mathbf{C} \hat{\mathbf{x}}),
$$

где $\hat{\mathbf{x}}$ - оценка вектора состояния $\mathbf{x} ; \mathbf{K}$ - матричный коэффициент обратной связи фильтра, задаваемый соотношением:

$$
\mathbf{K}=\mathbf{P C}^{T} \mathbf{R}^{-1} \text {; }
$$

$\mathbf{R}$ - матрица интенсивностей белого шума измерения; $\mathbf{P}$ - ковариационная матрица погрешности оценки вектора $\mathbf{x}$, определяемая из матричного дифференциального уравнения Риккати

$$
\mathbf{P}=\mathbf{A P}+\mathbf{P A}^{T}-\mathbf{P C}^{T} \mathbf{R}^{-1} \mathbf{C P} .
$$

Непосредственная реализация указанного алгоритма оценки состояния объекта наблюдения (10) по сигналу системы измерения (11) затруднена из-за того, что матрица $\mathbf{A}$ зависит от закона изменения угловой скорости объекта ориентации $\bar{\omega}$, а, кроме того, аналитическое решение уравнения Риккати (14) оказывается возможным лишь в ограниченном числе частных случаев, и поэтому, как правило, приходится использовать численные методы интегрирования для его решения.

В рассматриваемой задаче указанные трудности могут быть преодолены, прежде всего, путем проведения специальных калибровочных маневров космического аппарата, простейшим из которых является плоское вращение вокруг одной из осей связанного базиса $\mathbf{E}$ с постоянным визированием звездных ориентиров и определением ориентации космического аппарата в инерциальном координатном базисе.

Предположим, для определенности, что такое вращение производится вокруг третьей оси базиса $\mathbf{E}$. Тогда, очевидно, третья составляющая вектора $\bar{\alpha}$ по сигналу системы измерения (11) оказывается ненаблюдаемой, а две других составляющих этого вектора будут удовлетворять системе скалярных дифференциальных уравнений:

$$
\alpha \alpha=\omega_{z} \alpha_{2}, \alpha \alpha=-\omega_{z} \alpha_{1} \delta_{1}^{\alpha}=0, \delta_{2}^{\alpha}=0,
$$

где $\omega_{z}$ - угловая скорость выполнения калибровочного маневра, $\alpha_{1}, \alpha_{2}, \delta_{1}, \delta_{2}-$ соответствующие составляющие векторов $\bar{\alpha}$ и $\bar{\delta}$.

Уравнение системы измерения (11) при этом принимает вид:

$$
y_{1}=\alpha_{1}+\delta_{1}+\eta_{1}, y_{2}=\alpha_{2}+\delta_{2}+\eta_{2},
$$

где $y_{1}, y_{2}, \eta_{1}, \eta_{2}-$ составляющие векторов $\overline{\mathbf{y}}$ и $\bar{\eta}$ соответственно. 
Чтобы уравнения (15) были инвариантны относительно закона изменения угловой скорости вращения космического аппарата $\omega_{z}$, введем новую переменную $\varphi=\int_{9}^{t} \omega_{z}(\tau) d \tau$. Тогда уравнения (15) могут быть переписаны в виде:

$$
\alpha_{1}^{\prime}=\alpha_{2}, \alpha_{2}^{\prime}=-\alpha_{1} \delta_{1}^{\prime}=0, \delta_{2}^{\prime}=0 .
$$

(В уравнениях (17) штрихом обозначена производная по переменной $\varphi$ ).

Задача оценки состояния объекта наблюдения (17) по сигналам системы измерения (16) может быть решена на основании уравнений (12) - (14), в которых

$$
\mathbf{A}=\left\|\begin{array}{cccc}
0 & 1 & 0 & 0 \\
-1 & 0 & 0 & 0 \\
0 & 0 & 0 & 0 \\
0 & 0 & 0 & 0
\end{array}\right\|, \mathbf{C}=\left\|\begin{array}{cccc}
1 & 0 & 1 & 0 \\
0 & 1 & 0 & 1
\end{array}\right\|, \mathbf{R}=\left\|\begin{array}{cc}
r & 0 \\
0 & r
\end{array}\right\|
$$

( $r$ - интенсивность скалярного белого шума $\eta_{1}$ или $\eta_{2}$ ).

Для решения уравнения Риккати (14) целесообразно ввести замену переменной в соответствии с формулой

$$
\mathbf{P}=\mathbf{S}^{-1} \text {. }
$$

Используя правило дифференцирования обратной матрицы, вместо уравнения (14) будем иметь:

$$
-\mathbf{S}^{-1} \boldsymbol{S} \mathbf{S}^{-1}=\mathbf{A} \mathbf{S}^{-1}+\mathbf{S}^{-1} \mathbf{A}^{T}-\mathbf{S}^{-1} \mathbf{B}^{T} \mathbf{R}^{-1} \mathbf{B} \mathbf{S}^{-1},
$$

а умножая уравнение (19) на матрицу $\mathbf{S}$ слева и справа, окончательно получим:

$$
\S=-\mathbf{S A}-\mathbf{A}^{T} \mathbf{S}+\mathbf{B}^{T} \mathbf{R}^{-1} \mathbf{B} \text {. }
$$

Таким образом, уравнение Риккати (14) сводится к линейному матричному дифференциальному уравнению (20).

C учетом вида матриц $\mathbf{A}, \mathbf{C}, \mathbf{R}$ и симметричности матрицы $\mathbf{S}$ можно записать скалярную форму уравнения (20):

$$
\begin{aligned}
& s_{11}^{\prime}=-2 s_{12}+\frac{1}{r}, s_{12}^{\prime}=s_{11}-s_{22}, s_{13}^{\prime}=-s_{23}+\frac{1}{r}, s_{14}^{\prime}=-s_{24}, \\
& s_{22}^{\prime}=2 s_{12}+\frac{1}{r}, s_{23}^{\prime}=s_{13}, s_{24}^{\prime}=s_{14}+\frac{1}{r}, \\
& s_{33}^{\prime}=\frac{1}{r}, s_{34}^{\prime}=0, s_{44}^{\prime}=\frac{1}{r} .
\end{aligned}
$$

$\left(s_{i j}\right.$ - элементы матрицы $\left.\mathrm{S}, i, j=\overline{1,4}, j \geq i\right)$.

Система дифференциальных уравнений (21) декомпозируется на шесть взаимно независимых подсистем, каждая из которых допускает непосредственное интегрирование. Решение задачи Коши для системы (21) имеет вид:

$$
\begin{aligned}
& s_{11}(\varphi)=\frac{1}{2} s_{11}^{0}(1+\cos 2 \varphi)-s_{12}^{0} \sin 2 \varphi+\frac{1}{2} s_{22}^{0}(1-\cos 2 \varphi)+\frac{1}{r} \varphi, \\
& s_{12}(\varphi)=\frac{1}{2}\left(s_{11}^{0}-s_{22}^{0}\right) \sin 2 \varphi+s_{12}^{0} \cos 2 \varphi, \\
& s_{13}(\varphi)=s_{13}^{0} \cos \varphi-\left(s_{23}^{0}-\frac{1}{r}\right) \sin \varphi, \\
& s_{14}(\varphi)=\left(s_{14}^{0}+\frac{1}{r}\right) \cos \varphi-s_{24}^{0} \sin \varphi-\frac{1}{r}, \\
& s_{22}(\varphi)=\frac{1}{2} s_{11}^{0}(1-\cos 2 \varphi)+s_{12}^{0} \sin 2 \varphi+\frac{1}{2} s_{22}^{0}(1+\cos 2 \varphi)+\frac{1}{r} \varphi, \\
& s_{23}(\varphi)=s_{13}^{0} \sin \varphi+\left(s_{23}^{0}-\frac{1}{r}\right) \cos \varphi+\frac{1}{r}, \\
& s_{24}(\varphi)=\left(s_{14}^{0}+\frac{1}{r}\right) \sin \varphi+s_{24}^{0} \cos \varphi, \\
& s_{33}(\varphi)=s_{33}^{0}+\frac{1}{r} \varphi, s_{34}(\varphi)=s_{34}^{0}, s_{44}(\varphi)=s_{44}^{0}+\frac{1}{r} \varphi .
\end{aligned}
$$


где через $s_{i j}^{0}$ обозначены элементы матрицы начальных условий для матрицы $\mathbf{S}(i, j=\overline{1,4}, j \geq i)$.

Таким образом, выражения (22) позволяют вычислить значения элементов матрицы $\mathbf{S}$ как функции угла калибровочного поворота $\varphi$. Далее, в соответствие с (18), можно определить значения элементов матрицы $\mathbf{P}$, а затем, пользуясь уравнением (13), можно вычислить и матрицу коэффициентов обратной связи $\mathbf{K}$ оптимального линейного фильтра (12), который позволяет оценить составляющие $\alpha_{1}, \alpha_{2}, \delta_{1}, \delta_{2}$ векторов $\bar{\alpha}$ и $\bar{\delta}$.

Для определения остальных составляющих указанных векторов достаточно провести аналогичный калибровочный поворот космического аппарата вокруг другой оси связанного координатного базиса.

Полученное аналитическое решение задачи Коши для уравнения (14) позволяет осуществить параметрический синтез оптимального линейного фильтра Калмана-Бьюси. Кроме того, уравнение (14) может быть использовано для оценки предельно достижимой точности калибровки мультипликативных погрешностей измерителей угловой скорости при различных уровнях шумовой составляющей погрешности измерения.

\section{БИБЛИОГРАФИЧЕСКИЙ СПИСОК}

1. Дюмин А.Ф., Егоров С.Н., Суринский Д.М. Синтез устройств измерения угловой скорости космического аппарата//Космические исследования. 1994. Т. 32. Вып. 6. С. 50-65.

2. Егоров С.Н., Корабельщиков В.В., Суринский Д.М. Калибровка гироинерциальных измерителей систем ориентации по информации от неподвижных астровизиров//Известия АН СССР. МТТ. 1991. № 3. С. 7-12.

3. Дюмин А.Ф., Егоров С.Н., Корабельщиков В.В., Суринский Д.М. Способ калибровки гироинерциальных измерителей бесплатформенной инерциальной навигационной системы ориентации космического аппарата. Патент РФ № 2092402 от 10.10 .97$.

4. Дюмин А.Ф., Корабельщъиков В.В., Платонов С.Н., Суринский Д.М. Повышение точности астрокоррекции бесплатформенной инерциальной системы ориентации на электростатических гироскопах//Гироскопия и навигация. 2005. № 1. C. 76-83.

5. Бранеи В.Н. О точности решения кинематических уравнений//Космические исследования. 1982. Т. 20. Часть 1. Вып. 2. С. 184-190. Часть 2. Вып. 3. С. 323-331.

6. Андреев Ю.Н. Управление конечномерными линейными объектами. М.: Наука. 1976. 322 с.

7. Солодов А.В. Методы теории систем в задаче непрерывной линейной фильтрации. М.: Наука. 1976. 285 с. 